\title{
Vector-based Similarity Measurements for Historical Figures
}

\author{
Yanqing Chen, Bryan Perozzi, Steven Skiena \\ Dept. of Computer Science \\ Stony Brook University \\ Stony Brook, NY, 11794
}

\begin{abstract}
Historical interpretation benefits from identifying analogies among famous people: Who are the Lincolns, Einsteins, Hitlers, and Mozarts? As a knowledge source that benefits many applications in language processing and knowledge representation, Wikipedia provides the information we need to make such comparisons. We investigate several approaches to convert the Wikipedia pages of approximately 600,000 historical figures into vector representations to quantify similarity.

On the other hand, Wikipedia pages are assigned to different categories according to their contents as human-annotated labels. A rough similarity estimation could just counting the number of shared Wikipedia categories. However, such counting can neither make good similarity quantification (i.e. Is there a difference between those with same number of shared categories?) nor make distinguishable comments on different categories (i.e. Is US Presidents more important than state lawyer when defining similarity?). We use the counting as an indicator to demonstrate high-level agreements of our similarity detection algorithms.

In particular, we investigate four different unsupervised approaches to representing the semantic associations of individuals: (1) TF-IDF, (2) Weighted average of distributed word embedding, (3) LDA Topic analysis and (4) Deepwalk graph embedding from page links. All proved effective, but the Deepwalk embedding yielded an overall accuracy of $88.23 \%$ in our evaluation. Combining
\end{abstract}

Preprint submitted to Journal of ${ }^{A} T_{E} X$ Templates

April 25, 2016

C) 2015. This manuscript version is made available under the Elsevier user license http://www.elsevier.com/open-access/userlicense/1.0/ 
LDA and Deepwalk yielded even higher performance.

Finally, we demonstrate that our similarity measurements can also be used to recognize the most descriptive Wikipedia categories for historical figures. We rank the descriptive level of Wikipedia categories according to their categorical coherence, and our ranking yield an overall agreement of $88.27 \%$ compared with human crowdsourced data.

Keywords: Vector representations, People similarity, Deepwalk

\section{Introduction}

Historical interpretation benefits from identifying analogies among famous people: Who are the Lincolns, Einsteins, Hitlers, and Mozarts of today? Effective analogies should reflect shared personality traits, historical eras, and domains of accomplishment. Analogies are of course highly subjective, and rest at least partially in the eyes of the beholder: "there are a thousand Hamlets in a thousand people's eyes". Figure 1 provides an example, giving reasonable analogies on different aspects of Isaac Newton:

We are interested in developing a generalized model to identify analogous figures with a reasonably high similarity level, based on semantics in text and the connections of history. It could be very evocative if correctly identifying analogies like: Martin Luther King and Nelson Mandela; George Washington and Mao Zedong; Babe Ruth and Sachen Tendlukar.

In this paper, we propose methods for identifying historical analogies through the large-scale analysis of Wikipedia pages, as well as a reference standard to judge the effectiveness of our methods. The most obvious application of this is in historical interpretation and education, but we believe that the problem runs considerably deeper, since being able to identify similar individuals goes to the heart of algorithms for suggesting friends in social networks, or even matching algorithms pairing up roommates or romantic partners.

Specifically, our work makes the following contributions:

- We propose to use information extracted from Wikipedia categories to 


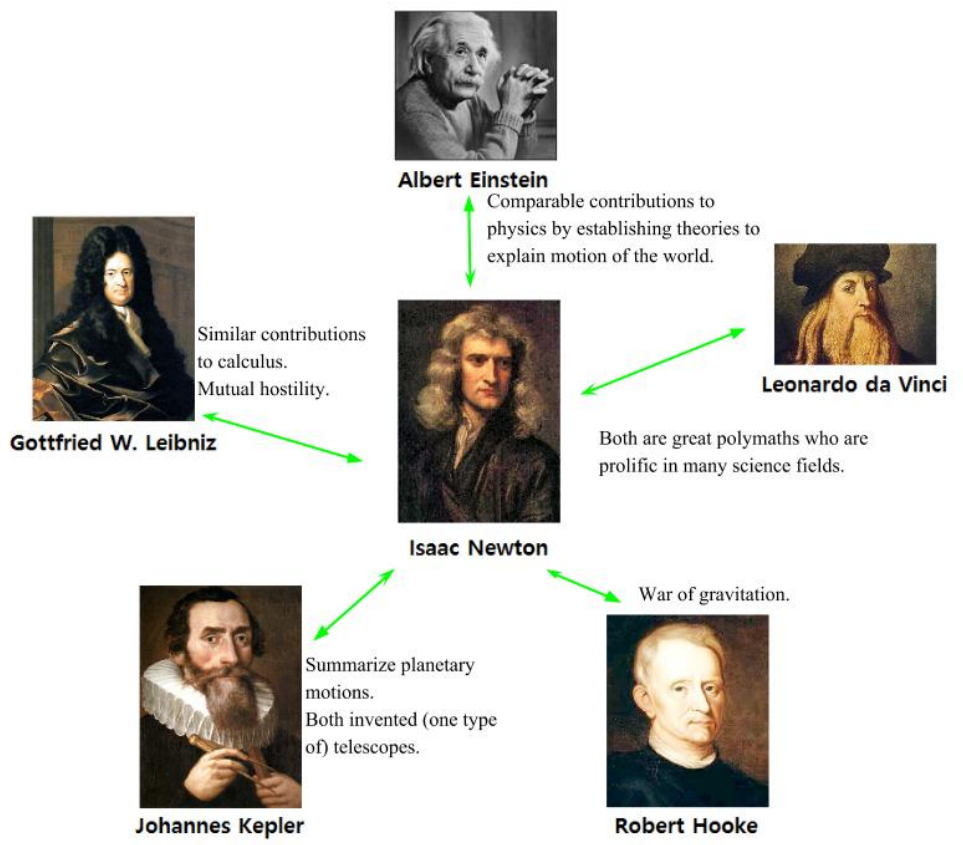

Figure 1: Analogous historical figures to Isaac Newton, with corresponding explanations of similarity. Analogies are highly subjective, making it impossible to find perfectly fair and objective gold standards.

be as reference standards to solve this task. Though not perfect, these human-labeled features imply relationships that are shared between similar people. We generated 3,000,000 triples of variable and prescribed difficulty, providing an effective standard to evaluate the performance of our similarity measurement algorithms.

- We investigate four different unsupervised approaches to extract semantic associations from Wikipedia. All proved effective, but our best approach with the graph embedding Deepwalk yielded an overall agreement of $88.23 \%$ with human annotated Wikipedia categories. We provide an interactive demonstration of our historical analogies at http://peoplesimilarity.appspot.com/, where you can identify the most similar historical figures to any queried 
individual.

- We did a careful search to identify the best distance function for each vector model. All metrics yield good results, but highlight different aspects of feature vectors. We also generated a model using linear combination of previously mentioned models to get a better tradeoff between graph structures and text semantics.

- We present a new approach to identifying the most salient categories associated with Wikipedia entities, based on the use of vector representations. We explore several different distance measures and coherence criteria to identify what is best at quantifying descriptive level of Wikipedia categories.

- Through human responses collected from Crowdflower, we verify that our notion of category appropriateness generally jibes with that of human reviewers. Indeed, we have fashioned an iOS game app (FameMatch, available on iTunes) testing how often users agree with our algorithmicallyselected categorization. We rank descriptive level of Wikipedia categories according to their coherence, which yield an overall agreement of $88.27 \%$ compared with human judgment.

This is an extended version of [1] with additional material:

- We emphasize the importance of "removing useless categories" by separating the experiment results to two components: before and after cleaning irrelevant categories. Counting the number of shared Wikipedia categories is not a good estimate unless we guarantee that the categories being counted are meaningful. We introduce ranking category importance in Section 6.

- We add additional introduction and commentary about four different types of vectorization, showing that these methods are intuitive and reasonable for our tasks. Each presents different advantages / disadvantages under different use cases. 
- We add several new sections presenting a follow-up experiment to rank Wikipedia category importance, and evaluating the performance through finding most important feature words of a page 5 . However, this approach still has some issues. One is that the probability distribution is not deterministic, especially when there are many closely-related topics. The other is that LDA focus only on co-occurrence of words, but a robust semantic grouping of topmakes the procedure semi-supervised. We will show later that unsupervised models can perform as well as, or even better than LDA on our tasks.

Learning vector representation of words or articles can help convert semantic features into high-dimensional spaces for easy quantification. Word-level repreembedding are able to perform well on several NLP tasks in the absence of any other features. Embedding with local information can lead to better and more precise clustering of words [9]. It is even possible to extract specific analogies of words from embedding [10, 11]. Additionally, word embedding can be easily a multilingual world. On sentence or article level, the proposed vector model 
in [13, 11] demonstrates the potential usage in related NLP tasks and 14] introduced a robust way of converting documents into vectors for a collection of corpus and keep those powerful features of embedding.

Network-based similarity measurements can be used for classification on large scale document collections [15], to provide unsupervised hierarchic similarity structure [16] and also to benefit visualizations to better understand "similarity" [17]. Deepwalk [18] seeks vector representations using recent advancements in language modeling and unsupervised feature learning (or deep learning) from sequences of words to graphs. Deepwalk uses truncated random walks to learn latent representations by encoding social relations in a continuous vector space that is easily exploited by statistical models. Applying Deepwalk on Wikipedia will create a vector representation for each page, thus providing a statistical comparison between entries in this huge graph.

There are many ways to evaluate our similarity measurements. One task is to retrieve real-life knowledge of reputations, fames and historical significance from entity ranking [19, and verify how well the vector representation can figure out the differences. Additionally, human annotated tags like Wikipedia categories also benefit many related tasks. Wikipedia categories can be used to boost search results in an ad hoc way [20. Researchers have shown that it is possible to analyze consistent semantic relationships in the tags [21, and the latent representations of raw text help tag recommendations [5]. Thus we believe an approach to rank Wikipedia categories based on latent representations of pages will better summarize the text content so as to provide better and more precise descriptions.

\section{Model Descriptions}

In this section we describe four candidate models which convert the content of a Wikipedia article to feature vectors to fuel similarity detection methods. These models are TF-IDF, distributed word embedding, LDA probability of topics, and Deepwalk embedding. 


\subsection{TF-IDF Model}

TF-IDF (short for Term FrequencyInverse Document Frequency) is a frequently used approach in NLP. Given a set of documents, TF computes raw frequency of each term in a document while IDF diminishes the weight of terms in the dictionary using deep-learning. The distances between vector representations measure differences in the meaning and usage of corresponding words. Several pre-computed word embedding are available online today, and SENNA is one which offers good performance on NLP tasks. The advantage of using that occur very frequently in the document set. The whole model captures the importance of words in target documents. One advantage of the TF-IDF model is that rare words are usually emphasized due to their uniqueness in the corpus, which makes it easy to handle words without pre-defined dictionary. We use the standard TF-IDF model in Gensim [22, so each Wikipedia people article document is converted to a feature vector that highlights the most important words with high TF-IDF value and a long tail of words with low TF-IDF value. We then compute pairwise similarity of documents using cosine similarity in vector space.

\subsection{Distributed Word Embedding Model}

Distributed word embedding learns a vector representation for each word word embedding is that syntactically and semantically similar words will be clustered together, to detect potential synonyms and improve the performance of bag-of-words processing. However the word embedding model has a disadvantage that it can only handle words with an existing embedding, so we will discard words not appearing in SENNAs dictionary. To compute the vector representation of an article, we calculate a weighted average embedding of the words in it. Our method is one type of implementation of doc2vec model. However, since our documents are not organically labeled, we have a different final target compared with Gensim doc2vec model.

By using TF-IDF value as weight for each word and combining the associated SENNA word embedding, feature vector of an article will be pulled to the 
barycenter of the most important and descriptive words in the article. Pairwise distance measurement in our experiment could be Euclidean distance, Manhattan distance or cosine similarity. Figure 2 is a simplified 2-D projection illustrating party leaders, musicians and physicists in our embedding space.



Figure 2: Sample entities and related words in projected embedding space. Entities are attracted by the most descriptive words in embedding space and located at the barycenter of these words, which can be used in distance calculation to show relevance between pairs.

LDA (short for Latent Dirichlet allocation) is a generative statistical model that allows sets of observations to be explained by unobserved groups. Calculation of LDA is based on the co-occurrence of words and assumes that each word will contribute to several topics with different weights. Documents are represented as a mixture of a small number of topics, so the LDA model outputs a probability distribution of corresponding topics as the feature vector for each document. We believe that correctly classified topics can further show similarities between documents, since in our case those documents and corresponding historical figures share achievements in similar fields. One advantage of LDA topic classification is that each topic is represented by words that contribute most to the topic, which makes it easy for human beings to understand 
and intuitively tell why two documents are similar. LDA is computationally demanding, so we run the LDA model for Wikipedia people corpus using a hyper-parameter of 500 topics. Figure 3 shows an example of how the topic distribution differs between party leaders, musicians and physicists.

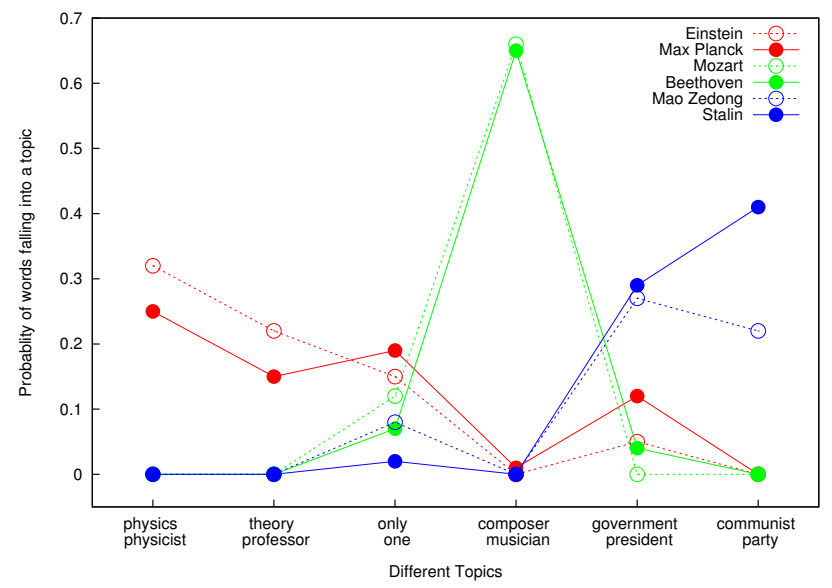

Figure 3: Examples of entities and related topics according to the LDA method. We display the two most representative words in each topic. The distribution of topics for Wikipedia people varies but historical figures with comparable contributions or related professional fields are more similar than the others.

We experiment with several metrics to measure similarity distance with the LDA vector, including previously used distance functions of Euclidean distance, Manhattan distance and cosine similarity. Additionally, KullbackLeibler divergence and JensenShannon divergence can be applied since feature vectors from LDA are probability distributions.

\subsection{Deepwalk Embedding Model}

Deepwalk creates statistical representations of graphs by learning from random walks on the graph and feature vectors are built from random-walk based representations of documents. Different from previous models, Deepwalk model does not rely on occurrences of words but only cares about links between documents. We propose that Wikipedia pages sharing more common links will sit closer in Deepwalk embedding space, since random walks in corresponding pages 
visit through very similar paths. Groups with a large fraction of links between corresponding Wikipedia people indicate stable relationships on similarity as random walks.

We use the package described in [18] with 128 output dimensions to train a Deepwalk embedding on adjacency lists of all Wikipedia pages (not just people's Wikipedia pages). These reference links work as a pre-defined set of topics to guide if two historical figures share similar historical achievements. This method requires link information to define a graph (i.e. may need extra co-reference resolution step from raw text), but it benefits from relatively confined collection of human knowledge like Wikipedia. This random walk method can easily be extended to multilingual world given that Wikipedia have different language versions. The distance function can be either Euclidean distance or Manhattan distance between Deepwalk embedding output of corresponding pages.

\section{Experimental Setup}

Wikipedia pages usually contain information from human-annotated categories such as eras, nationalities, occupations, awards and honors, educations, and important historical events. We believe that categories can construct memorable labels to indicate "similarities" and to remind us of the images of historical figures. Thus we adopt Wikipedia categories as reference standards to make distinctions between groups of people.

Let $F(X, Y)$ be the number of shared categories between entities $X$ and $Y$. We randomly select tuples of people $(X, Y, Z)$ with condition $F(X, Y)>$

${ }_{205} F(X, Z)$, indicating that $X$ shares more Wikipedia categories in common with $Y$ than with $Z$. Hence we declare that $Y$ is more similar to $X$ than $Z$ is.

We consider pair $(X, Y)$ to have high similarity if $F(X, Y) \geq 3$, while pair $(X, Y)$ has low similarity if $0<F(X, Y)<3$. Additionally, $F(X, Y)=0$ indicates zero similarity. We randomly sample 500,000 tuples of $(X, Y, Z)$ in each of the following three cases, ordered by difficulty level:

- Case I: High similarity vs. Zero: $F(X, Y) \geq 3$ and $F(X, Z)=0$ 
- Case II: Low similarity vs. Zero: $0<F(X, Y)<3$ and $F(X, Z)=0$

- Case III: High similarity vs. Low similarity: $F(X, Y) \geq 3$ and $0<F(X, Z)<3$ and $F(X, Y)-F(X, Z) \geq 2$

215

- Case IV: High similarity vs. High similarity: $F(X, Y) \geq 3$ and $F(X, Z) \geq 3$ and $F(X, Y)-F(X, Z) \geq 2$

Notices that Case III has an extra constraint to guarantee a statistical difference between two similarity levels. Case IV, the even harder one, works as a verification method to see how well Wikipedia categories replicate similarities when two historical figures are highly similar but showing differences on quantities of shared categories. We first construct 4 cases of experiments via random sampling using candidates from all 557,965 people. Then we build the similar testbed again but limit our candidates in the most famous 50,000 people according to significate score introduced in 19 to see if increasing overall number

of Wikipedia categories make a difference. Examples of our test bed are shown in Table 1 .

To evaluate, we measure how well our feature vectors agree with these test bed entries. We calculate distances between $X, Y$ and $X, Z$ for each tuple entry $(X, Y, Z)$ in our test bed. For a single tuple test, our feature vector passes 230 if distance between $X, Y$ is closer than distance between $X$ and $Z$. The final performance of each model is reported as the percentage of passed tuples tests among 3 test cases as well as the overall percentages.

\section{Results and Analysis}

Figure 4 shows the distribution of Wikipedia categories for all people's pages after preprocessing. The fame level is measured according to the ranking of significance in [19]. Famous people generally have many more Wikipedia categories than less significant people, which is why we cannot measure people similarity based only on these category descriptions. We build up our reference standard 


\begin{tabular}{|c|l|}
\hline Case & (X, Y, Z) \\
\hline I & $\begin{array}{l}\text { (Einstein, Oppenheimer, Michael Jackson) } \\
\text { (Lincoln, Reagan, Gaddafi) } \\
\text { (Mozart, Brahms, Michael Phelps) }\end{array}$ \\
\hline II & $\begin{array}{l}\text { (Einstein, Aristotle, Celine Dion) } \\
\text { (Lincoln, Bill Clinton, Heath Ledger) } \\
\text { (Mozart, Charlie Chaplin, Larry Bird) }\end{array}$ \\
\hline III & $\begin{array}{l}\text { (Einstein, Richard Feynman, John Jacob Abel) } \\
\text { (Lincoln, Ulysses Grant, George W. Bush) } \\
\text { (Mozart, Beethoven, Dmitri Shostakovich) }\end{array}$ \\
\hline IV & $\begin{array}{l}\text { (Einstein, Hans Bethe, Yoichiro Nambu) } \\
\text { (Lincoln, James A. Garfield, Harold Washington) } \\
\text { (Mozart, Franz Schubert, Georg Joseph Vogler) }\end{array}$ \\
\hline
\end{tabular}

Table 1: Tuple examples (X, Y, Z) in 3 different cases. Pair (X, Y) is always closer than Pair $(\mathrm{X}, \mathrm{Z})$ in our tuples. Cases are sorted according to difficulty so making correct judgments on Case III and Case IV is harder.

according to the number of categories shared between two people, assuming that the more categories shared implies the more similar the people are.

We first tested our models against count of common Wikipedia categories. Unfortunately the results did not prove illuminating, - with poor performance (e.g. accuracy close to $50 \%$ ) which did not distinguish between models. We discovered that certain categories provide insignificant information for our task, such as "Living people" or "Born in 1957". In order to create a better testbed, we did preprocessing to find out categories that are too broad to be interesting, including "living people", "year of birth / death" and "Birthplace". We eliminated all categories which matched these patterns and ran our experiment again.

Table 2 gives the accuracy of four models under five different distance metrics, on tests over all people in Wikipedia and then restricted to the 50,000 most famous people. The accuracy now shows that our similarity metrics really tell 


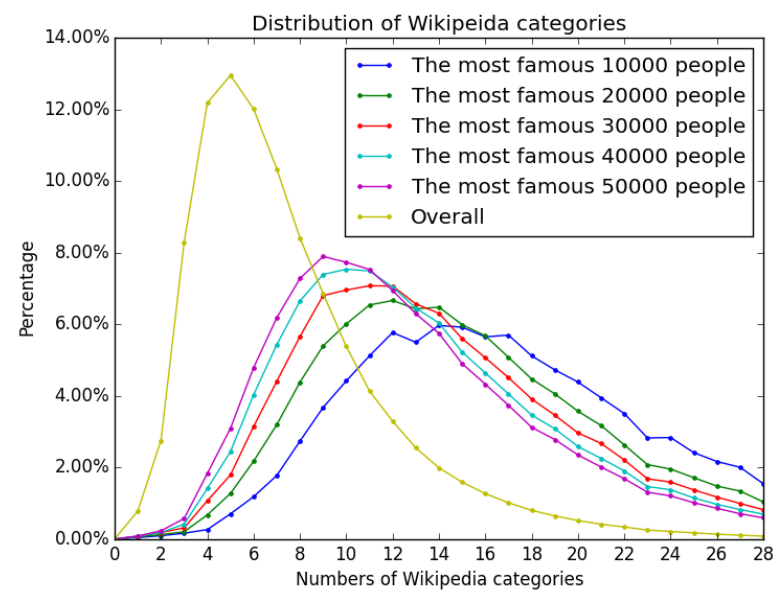

Figure 4: Distribution of Wikipedia categories on pages of people. The most detailed information usually coincides with the most famous historical figures, as demonstrated by increased number of Wikipedia categories.

the difference and we believe there should be some category ranking work to benefit our similarity tasks, which will be introduced in later.

The TF-IDF model answers $77.50 \%$ of the questions correctly, much better than the baseline of random guessing ( $50 \%$ accuracy). This shows that the most important words can induce similarity well. However, TF-IDF has no knowledge of syntactic changes in words (e.g. great vs greatest) and synonyms (e.g. emperor vs monarch). Study of the error cases shows that TF-IDF focuses too much on locations and names, which reduces its ability to recognize more important words in similarity measurement. The word embedding model clusters synonyms in embedding space, so the feature vectors capture topic words with similar semantic meaning, resulting in better performance compared against the original TF-IDF. However, it is hard for TF-IDF model to correctly answer questions in Case IV, as the difference between feature vectors are not enough to quantify similarity.

The LDA model performs well on Case I and Case II instances compared to other models. However in Case III, where entities have shared categories in our 


\begin{tabular}{|c|c|c|c|c|c|c|}
\hline \multicolumn{7}{|c|}{ Overall } \\
\hline Model & Distance & Case I & Case II & Case III & Case IV & Overall \\
\hline TF-IDF & Cosine & $87.01 \%$ & $76.23 \%$ & $77.98 \%$ & $68.79 \%$ & $77.50 \%$ \\
\hline \multirow{3}{*}{ Word embedding } & L2 & $96.95 \%$ & $84.40 \%$ & $74.97 \%$ & $65.66 \%$ & $80.50 \%$ \\
\hline & L1 & $96.56 \%$ & $84.26 \%$ & $75.57 \%$ & $66.34 \%$ & $80.69 \%$ \\
\hline & Cosine & $96.43 \%$ & $84.13 \%$ & $75.45 \%$ & $66.81 \%$ & $80.71 \%$ \\
\hline \multirow{5}{*}{ LDA } & L2 & $98.70 \%$ & $88.22 \%$ & $75.39 \%$ & $67.14 \%$ & $82.36 \%$ \\
\hline & L1 & $98.17 \%$ & $88.35 \%$ & $77.26 \%$ & $69.77 \%$ & $83.39 \%$ \\
\hline & Cosine & $98.43 \%$ & $88.60 \%$ & $77.53 \%$ & $70.01 \%$ & $83.64 \%$ \\
\hline & $\mathrm{KL}$ & $97.57 \%$ & $87.86 \%$ & $76.10 \%$ & $70.12 \%$ & $82.91 \%$ \\
\hline & JS & $97.69 \%$ & $87.98 \%$ & $76.22 \%$ & $70.09 \%$ & $83.00 \%$ \\
\hline \multirow{2}{*}{ Deepwalk } & L2 & $99.51 \%$ & $89.50 \%$ & $84.97 \%$ & $78.95 \%$ & $88.23 \%$ \\
\hline & L1 & $99.11 \%$ & $89.13 \%$ & $84.59 \%$ & $77.82 \%$ & $87.66 \%$ \\
\hline \multicolumn{7}{|c|}{ Most Famous 50,000 people } \\
\hline Model & Distance & Case I & Case II & Case III & Case IV & Overall \\
\hline TF-IDF & Cosine & $87.88 \%$ & $77.06 \%$ & $78.37 \%$ & $70.50 \%$ & $78.45 \%$ \\
\hline \multirow{3}{*}{ Word embedding } & L2 & $96.89 \%$ & $82.92 \%$ & $90.23 \%$ & $71.74 \%$ & $85.44 \%$ \\
\hline & L1 & $96.51 \%$ & $82.90 \%$ & $89.04 \%$ & $71.59 \%$ & $85.01 \%$ \\
\hline & Cosine & $96.31 \%$ & $82.68 \%$ & $88.85 \%$ & $71.43 \%$ & $84.82 \%$ \\
\hline \multirow{5}{*}{ LDA } & L2 & $97.68 \%$ & $83.71 \%$ & $80.99 \%$ & $71.55 \%$ & $83.48 \%$ \\
\hline & L1 & $97.95 \%$ & $83.31 \%$ & $81.26 \%$ & $70.96 \%$ & $83.37 \%$ \\
\hline & Cosine & $97.63 \%$ & $83.65 \%$ & $80.94 \%$ & $71.22 \%$ & $83.36 \%$ \\
\hline & $\mathrm{KL}$ & $96.86 \%$ & $83.58 \%$ & $80.84 \%$ & $71.49 \%$ & $83.19 \%$ \\
\hline & JS & $97.15 \%$ & $83.89 \%$ & $81.14 \%$ & $72.01 \%$ & $83.54 \%$ \\
\hline \multirow{2}{*}{ Deepwalk } & L2 & $98.73 \%$ & $85.47 \%$ & $91.59 \%$ & $82.51 \%$ & $89.58 \%$ \\
\hline & L1 & $98.11 \%$ & $84.85 \%$ & $90.79 \%$ & $80.95 \%$ & $88.68 \%$ \\
\hline
\end{tabular}

Table 2: Accuracy performance of candidate models after cleaning useless categories. LDA model has extra metrics since the output feature vectors are probability distributions. Deepwalk slightly outperforms LDA. 
references, LDA failed to capture the similarity from topic distributions. This phenomenon indicates that we may need to control topic numbers to limit the detail level in our task. Additionally, we found that distance metrics do not lead to huge performance improvements in LDA, showing LDA feature vectors are robust and well-shaped in embedding space, reflecting good hierarchical similarity structure.

The Deepwalk embedding yielded an overall accuracy of $88.23 \%$ on the allpeople test and an even higher $91.93 \%$ on 50,000 most famous people test, beating all other vector based models. The noticeable drop in Case II accuracy is caused by "weak" categories that do not provide strong support on similarity measurement. Another discovery is that important historical events will usually pull people closer in Deepwalk embedding space.

Though we consider Case III (quantification of similarities) to be more difficult than Case II (detecting minor similarities), the TF-IDF model (as well as Deepwalk and Distributed word embedding model when targeting most famous 50,000 Wikipedia figures) answers Case III questions more accurately.

We did a manual human evaluation on 200 randomly-selected people using LDA, Deepwalk and linear combination of both. Result shows that text-based methods like LDA plays complementary roles to Deepwalk since these two approaches emphasize different part in similarity. Agreement from both methods undoubtedly increase the confidence level of quantifying similarity. Table 3 shows ten examples and corresponding analogous historical figures, as well as human evaluations using single or combined distance measurement from our previous experiments.

\section{Identifying Categorical Importance}

Wikipedia pages describing important historical figures are usually assigned to many categories. Here we evaluate whether our vector representations can be used to distinguish the most important categories from mediocre ones. For example, Barack Obama is listed in almost fifty different Wikipedia categories, 


\begin{tabular}{|c|c|c|c|c|c|c|c|c|c|}
\hline \multirow{2}{*}{ Model } & \multicolumn{3}{|c|}{ Albert Einstein } & \multicolumn{3}{|c|}{ Yao Ming } & \multicolumn{3}{|c|}{ Larry Page } \\
\hline & Candidates & $\mathrm{C}$ & $\mathrm{HE}$ & Candidates & $\mathrm{C}$ & $\mathrm{HE}$ & Candidates & $\mathrm{C}$ & $\mathrm{HE}$ \\
\hline \multirow{10}{*}{ LDA } & Wolfgang Pauli & 5 & ++ & Yi Jianlian & 4 & ++ & Simson Garfinkel & 0 & ++ \\
\hline & Emmy Noether & 1 & - & Chris Bosh & 2 & ++ & Robert Metcalfe & 1 & ++ \\
\hline & Erwin Schrdinger & 2 & ++ & Sun Yue & 3 & ++ & John Mashey & 0 & - \\
\hline & Eugene Wigner & 4 & ++ & Luol Deng & 1 & + & Ray Tomlinson & 0 & - \\
\hline & Norbert Wiener & 1 & - & Bob Cousy & 1 & - & M. J. Dominus & 0 & - \\
\hline & Esther Lederberg & 0 & - & Steve Nash & 2 & + & R. Piquepaille & 0 & - \\
\hline & David Hilbert & 0 & + & Herschel Walker & 0 & - & Ellen Spertus & 1 & - \\
\hline & Felix Ehrenhaft & 0 & + & R. Tomjanovich & 2 & + & Jon Lebkowsky & 0 & + \\
\hline & Paul Ehrenfest & 1 & + & A. Kavaliauskas & 1 & + & R. P. Garbriel & 2 & ++ \\
\hline & Ralph Kronig & 1 & + & Mengke Bateer & 5 & ++ & D. Giampaolo & 1 & + \\
\hline \multirow{10}{*}{ Deepwalk } & Richard Feynman & 4 & ++ & Yi Jianlian & 4 & ++ & Sergey Brin & 12 & ++ \\
\hline & Max Planck & 3 & ++ & Jeremy Lin & 0 & ++ & Eric Schmidt & 6 & ++ \\
\hline & Freeman Dyson & 3 & + & Kobe Bryant & 2 & + & Bill Gates & 6 & ++ \\
\hline & David Bohm & 1 & + & Wang Zhizhi & 5 & ++ & Marc Andreessen & 2 & ++ \\
\hline & Stephen Hawking & 2 & ++ & Michael Jordan & 1 & + & Mark Zuckerberg & 6 & ++ \\
\hline & David Hilbert & 0 & + & Deron Williams & 2 & + & Esther Dyson & 0 & - \\
\hline & Oppenheimer & 4 & ++ & Mengke Bateer & 5 & ++ & John Doerr & 3 & + \\
\hline & Werner Heisenberg & 4 & ++ & Dwyane Wade & 3 & + & John Battelle & 0 & ++ \\
\hline & Hermann Bondi & 1 & - & LeBron James & 3 & + & Joi Ito & 0 & - \\
\hline & Erwin Schrdinger & 2 & ++ & Steve Francis & 2 & + & Jimmy Wales & 1 & + \\
\hline \multirow{2}{*}{ Linear } & Max Planck & 3 & ++ & Yi Jianlian & 4 & ++ & Bill Gates & 6 & ++ \\
\hline & Erwin Schrdinger & 2 & ++ & Mengke Bateer & 5 & ++ & Eric Schmidt & 6 & ++ \\
\hline \multirow{2}{*}{ comb } & Richard Feynman & 4 & ++ & Chris Bosh & 2 & ++ & Simson Garfinkel & 0 & ++ \\
\hline & Freeman Dyson & 3 & + & Michael Jordan & 1 & + & Sergey Brin & 12 & ++ \\
\hline \multirow{2}{*}{ of } & Wolfgang Pauli & 5 & ++ & LeBron James & 3 & + & Robert Metcalfe & 1 & ++ \\
\hline & David Bohm & 1 & + & Jeremy Lin & 0 & ++ & Marc Andreessen & 2 & ++ \\
\hline \multirow{2}{*}{ Deepwalk } & Eugene Wigner & 4 & ++ & Charles Barkley & 2 & ++ & Mark Zuckerberg & 6 & ++ \\
\hline & R. Millikan & 2 & + & Tony Parker & 1 & + & John Battelle & 0 & ++ \\
\hline \multirow{2}{*}{ and LDA } & Stephen Hawking & 2 & ++ & Steve Francis & 2 & + & Marissa Mayer & 4 & + \\
\hline & George Gamow & 2 & + & Juwan Howard & 2 & ++ & Steve Jobs & 5 & ++ \\
\hline
\end{tabular}

Table 3: Examples of the ten closest neighbors we find using our vector based models and corresponding human evaluations. The $C$ column counts the common Wikipedia categories between pairs of people, and the $H E$ column presents a human evaluation after reading their bibliography with,+++ or - rating the quality of the analogy.

including " 20th-century American writers", "1961 births", "American Nobel laureates", "Grammy Award winners" and "Harvard Law School alumni". 300 These categories are undoubtedly correct but generally irrelevant. The cat- 
egory "Presidents of the United States" best captures his historical/cultural significance.

One possible approach to evaluate categories is based on keywords, e.g. recognizing that "President" is a more important distinction than other titles. However, this appears quite challenging. Being president of a small organization is generally less impressive than a lesser position in an important organization. Thus, evaluating the magnitude of such accomplishments based on keywords seems difficult and subjective.

Instead, we propose to recognize the most descriptive Wikipedia categories based on categorical coherence. We observe that the figures in a precise category should be mutually similar: the presidents of the United States should more closely resemble each other than "American writers" or "Grammy Award" recipients. Such categorical coherence can be evaluated via similarities calculated from our vector representations, and we believe an interesting category requires coherence to make it interesting. We rank the descriptive level of Wikipedia categories according to their coherence, which yield an overall agreement of $88.27 \%$ compared with a crowdsourced gold standard.

\subsection{Evaluation Standard}

To build a gold standard for categorical accuracy, we employed Crowdflower, a leading people-powered data enrichment platform. We solicited human reviews of the most descriptive Wikipedia categories on the 500 most significant people as described in [19. For each Wikipedia person we manually selected four categories with good descriptive power, plus an additional random category to make up a question with five choices.

325

Each user selection clarifies which single category choice dominates the other four for a particular subject. By asking each question to 20 reviewers, the categories with higher descriptive power will accumulate more votes, so the distribution indicates the overall descriptive power of each Wikipedia category. In total we gathered 10,000 responses from 176 volunteers, covering 1076 distinct Wikipedia categories. 


\section{Experiments on Category Ranking}

In this section we describe how we calculate categorical coherence. The procedure involves four major steps: (1) generating feature vectors using latent representations from text or links; (2) measuring distances in vector space; (3)

335 on observations of close neighbors. We will have multiple possibilities to implement each step in the experiment and we seek the best combinations to solve this task.

\subsection{Generating feature vectors}

340 including LDA and Deepwalk [18] with 128 output dimensions trained on the adjacency lists of all Wikipedia pages.

\subsection{Measuring distances}

Feature vectors are usually considered as points in high-dimensional space. ization), or Euclidean distance (L2 normalization), and cosine similarity to measure similarities between feature vectors.

Additionally, since LDA represents probability distributions, we include KullbackLeibler divergence and Jensen-Shannon divergence in our distance metrics.

\subsection{Definition of close neighbors}

Distances between feature vectors indicate similarity between corresponding Wikipedia people. However, twice the distance does not necessarily mean half the similarity. On the other hand, observation shows that only pairs within a certain range show strong signals of similarity. Such ranges are usually correlated with the density of feature vectors in a certain area of the embedding space. 
We propose two ways to define nearby neighbors. One limits the count, considering only the $K$ closest neighbors in vector space to be "close". The relationship between close neighbors is not always reversible under this definition, so this strategy can create asymmetric results. The other approach picks neighbors by distance, identifying the points within a threshold distance of $D$. Under this strategy, semi-isolated points may have no points identified similar to it. Both approaches will be considered as a hyper-parameter in our experiment.

\subsection{Ranking criteria}

365 We propose two methods to quantify how descriptive a Wikipedia category is: conductance and surprise level.

\subsubsection{Conductance}

The conductance of a category is defined as the ratio between close neighbors inside the category and those outside the category. Categories with more close neighbors inside should be more stable, since neighbors share similarities with internal members. However, this method ignores the size of the category. Larger categories with more people become more diverse, thus reducing the conductance of the category.

Let $C_{c a t}$ be the conductance of a category, then:

$$
C_{\text {cat }}=\sum_{\substack{X \in \text { cat } \\ Y \in \text { cat } \\(X, Y) \in \text { closeneighbors }}} / \sum_{\substack{X \in \text { cat } \\(X, Y) \in \text { closeneighbors }}}
$$

\subsubsection{Surprise level}

The other measurement we consider is surprise level. This metric focuses on balancing the effect of both size of the category and the probability that inside-category pairs become close neighbors. We assume that the probability that "a pair is inside a category" is independent from the probability of "a pair shares a close-neighbor relationship". Then for an entity $A$ in the category the probability of its close neighbor $B$ belongs to the category will be:

$$
P_{\text {cat }}=\sum_{X \in c a t} / \sum_{X}
$$




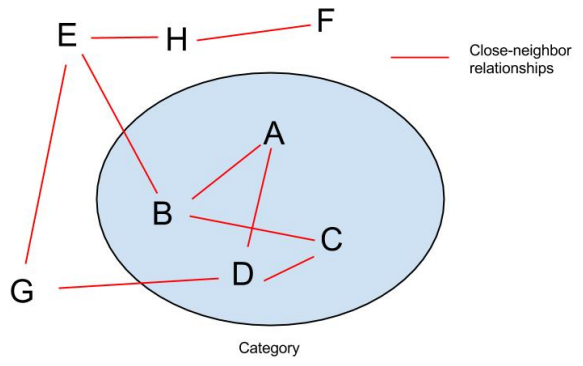

Figure 5: The method of calculating conductance. The category has four entities and four internal close-neighbor relationships. However, there are two entities (E, G) which are close to one of the inside members with a total of three close-neighbor relationships that are outside the category. The conductance of this category is $\frac{4}{4+2} \approx 0.6667$. $K$ close neighbors in the group will be:

$$
P\left(\text { cat }, K, C_{A}\right)=\left(\begin{array}{c}
C_{A} \\
K
\end{array}\right)\left(P_{c a t}^{K}\left(1-P_{c a t}\right)^{C_{A}-K}\right)
$$

The surprise level of the category $G_{A}$ from observer $A$ is defined as:

$$
S_{c a t, A}=\sum_{X \geq G_{A}} P\left(\text { cat }, X, C_{A}\right)
$$

We then average the surprise level for all observers in the category to get the categorical surprise level. The example in Figure 5 results in following values:

$$
\begin{gathered}
P_{c a t}=\frac{4}{8}=0.5 \\
S_{c a t, A}=S_{c a t, C}=\left(\begin{array}{l}
2 \\
2
\end{array}\right) P_{c a t}=0.25 \\
S_{c a t, B}=S_{c a t, D}=\left(\begin{array}{l}
3 \\
2
\end{array}\right) P_{c a t}+\left(\begin{array}{l}
3 \\
3
\end{array}\right) P_{c a t}=0.5 \\
S_{c a t}=\frac{0.25+0.5+0.25+0.5}{4}=0.375
\end{gathered}
$$




\subsection{Experimental Setup}

In our experiment, we conducted a grid search with following choices:

- Feature vectors: (LDA, Deepwalk)

- Distance function: (L1, L2, Cosine, KL (for LDA) and JS (for LDA))

- Close neighbors: (Count and Distance). For count strategy we will test using parameters of 5, 10, 25, 50 and 100. For distance strategy we sort pair-wise distances and choose thresholds to keep an average of $5,10,25$, 50 and 100 close neighbors correspondingly.

- Measurement: (conductance, surprise level)

\section{Evaluation results and analysis}

We evaluate the quality of our ranking according to agreement with human annotations, i.e. the ranks of 1067 categories with crowdsourced annotations.

\subsection{Basic evaluation}

A single response in our crowdsourced data identifies the most descriptive category from among five possible choices. Thus each response actually indicates four binary comparisons between the selected one and the others. If the human response ranks first by our method, we award it one point for making four correct judgments. In general, we score $(5-i) \times 0.25$ points where $i$ is the relative rank among the five choices in our ranking. Thus when the human response ranks second, we score it as 0.75 points. The rough overall accuracy is calculated as:

$$
\text { Acc }_{\text {rough }}=\frac{\sum_{i} \text { Score for answer }}{\text { answers } \mid}
$$

where the total number of answers is 500 questions * 20 answers each question $=10,000$. 
Our human reviews are not perfectly consistent, and we observe substantial conflicts between their answers. Observations on the data show that certain categories proved highly confusing, because they describe highly-intersecting sets of people. Table 4 lists the ten most confusing category pairs.

\begin{tabular}{|l|c|}
\hline Category pairs & Co-Prob \\
\hline French Open champions & $100.00 \%$ \\
\hline Wimbledon champions & $100.00 \%$ \\
\hline Australian film actors & \\
Australian television actors & $86.60 \%$ \\
\hline $\begin{array}{l}\text { Association football forwards } \\
\text { Brazilian footballers }\end{array}$ & $86.60 \%$ \\
\hline $\begin{array}{l}\text { Holocaust perpetrators } \\
\text { Nazi Germany ministers }\end{array}$ & $81.65 \%$ \\
\hline $\begin{array}{l}\text { National Basketball Association All-Stars } \\
\text { Parade High School All-Americans (boys' basketball) }\end{array}$ & $67.36 \%$ \\
\hline $\begin{array}{l}\text { Eastern Orthodox saints } \\
\text { People celebrated in the Lutheran liturgical calendar }\end{array}$ & $75.59 \%$ \\
\hline American novelists & $67.94 \%$ \\
\hline American short story writers & $62.90 \%$ \\
\hline $\begin{array}{l}\text { American jazz singers } \\
\text { Traditional pop music singers }\end{array}$ & \\
\hline American rhythm and blues singer-songwriters & \\
\hline American soul singers & \\
\hline
\end{tabular}

Table 4: The ten most confusing/ambiguous category pairs in our questions. The Co-Prob of two categories $A$ and $B$ is defined as the geometric mean of $P(A \mid B)$ and $P(B \mid A)$.

Because of these conflicting annotator selections, the best theoretical performance of any system will be substantially lower than $100 \%$. To calculate the optimal performance possible on this task, we construct a graph whose nodes 
are Wikipedia categories with directed edges to indicate human responses that $a$ is better than $b$. We run a topological sort algorithm to identify the best achievable score from the Crowdflower human reviews, indicating that in any system at least $15.5 \%$ answers must be incorrect.

The improved overall accuracy measure is now set to:

$$
\text { Acc }_{\text {improved }}=\frac{\sum_{i} \text { Score for answer }_{i}}{\mid \text { BestPossibleScore } \mid}
$$

\subsection{Influence of feature vectors}

We first discuss the influence of different feature vectors in Table 5. Deepwalk basically outperforms LDA, getting $1.5 \%$ more answers correct. The distance distribution of both vectors are stable since the gap between each threshold ( 5 , $10,25,50,100)$ is increasing, indicating that points in high-dimensional spaces are not too dense. Deepwalk also wins in the mean performances among all parameters choices, with a higher accuracy and lower standard deviation.

\subsection{Influence of distance measurement}

Table 6 shows statistics of how different distance measurements change the performance.

From our observations there are no big differences in best/average performance between L1 normalization and L2 normalization. Cosine similarity works well on average cases but it does not win to produce the best performance. KL divergence and JS divergence, though specifically designed for probability distributions, do not yield better performances in our tasks. This phenomena probably indicates the redundancy in our feature vectors, so that no matter which normalization function was chosen, there will be a feature that preserves making similar points close enough.

\subsection{Influence of defining close neighbors}

Figure 6 shows ten different definitions of similarity, with corresponding performances, including five using count as threshold and five using distance as threshold. 


\begin{tabular}{|c|c|c|c|c|}
\hline & & & \multicolumn{2}{|c|}{ Feature vector } \\
\hline & & & LDA & Deepwalk \\
\hline \multirow{15}{*}{ Best } & \multicolumn{2}{|c|}{ Overall Accuracy } & $86.70 \%$ & $88.27 \%$ \\
\hline & \multirow{4}{*}{ Parameters } & Distance & L1 & L2 \\
\hline & & Closeness & Count & Count \\
\hline & & Avg Neighbors & 25 & 50 \\
\hline & & Ranking & SL & SL \\
\hline & \multirow{5}{*}{ Agreement } & 1st & $44.07 \%$ & $46.47 \%$ \\
\hline & & $2 \mathrm{nd}$ & $25.10 \%$ & $24.79 \%$ \\
\hline & & $3 \mathrm{rd}$ & $16.29 \%$ & $14.97 \%$ \\
\hline & & 4 th & $9.11 \%$ & $8.32 \%$ \\
\hline & & 5 th & $5.44 \%$ & $5.44 \%$ \\
\hline & \multirow{5}{*}{ Distance } & 5 & 0.1352 & 1.0092 \\
\hline & & 10 & 0.1972 & 1.0704 \\
\hline & & 25 & 0.3091 & 1.1740 \\
\hline & & 50 & 0.4223 & 1.2823 \\
\hline & & 100 & 0.5639 & 1.4366 \\
\hline \multirow{7}{*}{ Average } & \multicolumn{2}{|c|}{ Overall Accuracy } & $75.67 \%$ & $78.01 \%$ \\
\hline & \multicolumn{2}{|c|}{ Std Deviation } & 0.062 & 0.044 \\
\hline & \multirow{5}{*}{ Agreement } & 1 st & $33.55 \%$ & $36.67 \%$ \\
\hline & & 2nd & $23.86 \%$ & $22.81 \%$ \\
\hline & & $3 \mathrm{rd}$ & $18.13 \%$ & $17.51 \%$ \\
\hline & & 4 th & $13.93 \%$ & $13.71 \%$ \\
\hline & & 5 th & $10.53 \%$ & $9.30 \%$ \\
\hline
\end{tabular}

Table 5: Performance of each feature vector on category ranking. Best rows describe the best achieved performance, including improved overall accuracy, model parameters (distance measurement, close neighbor definition, average count close neighbors and ranking criteria), distribution of points gaining and corresponding distance distribution in vector spaces. $A v$ erage rows provide information of average accuracy, standard deviation and distribution of average points gaining. 


\begin{tabular}{|c|c|c|c|c|c|c|c|}
\hline & & & \multicolumn{5}{|c|}{ Distance measurements } \\
\hline & & & L1 & L2 & Cos & $\mathrm{KL}$ & JS \\
\hline \multirow{6}{*}{ Best } & \multicolumn{2}{|c|}{ Accuracy } & $87.94 \%$ & $88.27 \%$ & $86.70 \%$ & $85.73 \%$ & $86.45 \%$ \\
\hline & \multirow{5}{*}{ Agree } & $1 \mathrm{st}$ & $46.09 \%$ & $46.47 \%$ & $44.07 \%$ & $43.71 \%$ & $43.99 \%$ \\
\hline & & 2nd & $24.91 \%$ & $24.79 \%$ & $25.10 \%$ & $24.73 \%$ & $24.12 \%$ \\
\hline & & $3 r d$ & $14.91 \%$ & $14.97 \%$ & $16.29 \%$ & $14.25 \%$ & $17.33 \%$ \\
\hline & & 4 th & $8.57 \%$ & $8.32 \%$ & $9.11 \%$ & $12.45 \%$ & $9.41 \%$ \\
\hline & & 5 th & $5.52 \%$ & $5.44 \%$ & $5.14 \%$ & $4.87 \%$ & $5.14 \%$ \\
\hline \multirow{7}{*}{ Average } & \multicolumn{2}{|c|}{ Accuracy } & $75.41 \%$ & $77.94 \%$ & $78.87 \%$ & $75.98 \%$ & $76.10 \%$ \\
\hline & \multicolumn{2}{|c|}{ Std Deviation } & 0.062 & 0.044 & 0.033 & 0.063 & 0.063 \\
\hline & \multirow{5}{*}{ Agree } & $1 \mathrm{st}$ & $33.51 \%$ & $36.53 \%$ & $37.07 \%$ & $34.03 \%$ & $33.96 \%$ \\
\hline & & 2nd & $23.53 \%$ & $22.93 \%$ & $22.78 \%$ & $23.50 \%$ & $23.85 \%$ \\
\hline & & $3 r d$ & $18.18 \%$ & $17.48 \%$ & $18.38 \%$ & $18.30 \%$ & $18.19 \%$ \\
\hline & & 4 th & $14.06 \%$ & $13.77 \%$ & $13.37 \%$ & $13.77 \%$ & $13.63 \%$ \\
\hline & & 5 th & $10.71 \%$ & $9.29 \%$ & $8.40 \%$ & $10.39 \%$ & $10.36 \%$ \\
\hline
\end{tabular}

Table 6: Performance of each distance measurement. JS and KL are uniquely used in LDA model. Best rows describe the best achieved performance over all possible parameter choices. Average rows provide information of average accuracy, standard deviation and distribution of average points gaining.

It is clear that judging close neighbors using only the value of distance makes it worse. The reason is that the density of isolated points is much lower than for frequently mentioned historical figures. Setting the threshold to be a certain diameter will create uneven distribution of close neighbors, and thus lower the quality and stability of similarity measurement.

Both count and distance thresholds show a peak within the range of 25 to 50 close neighbors, which is approximately $0.005 \%$ to $0.01 \%$ of the 557,596 Wikipedia pages under consideration. Neither increasing nor decreasing this value give better performance. We believe such criteria would still work even under more sophisticated circumstances, e.g. considering most famous 50,000 


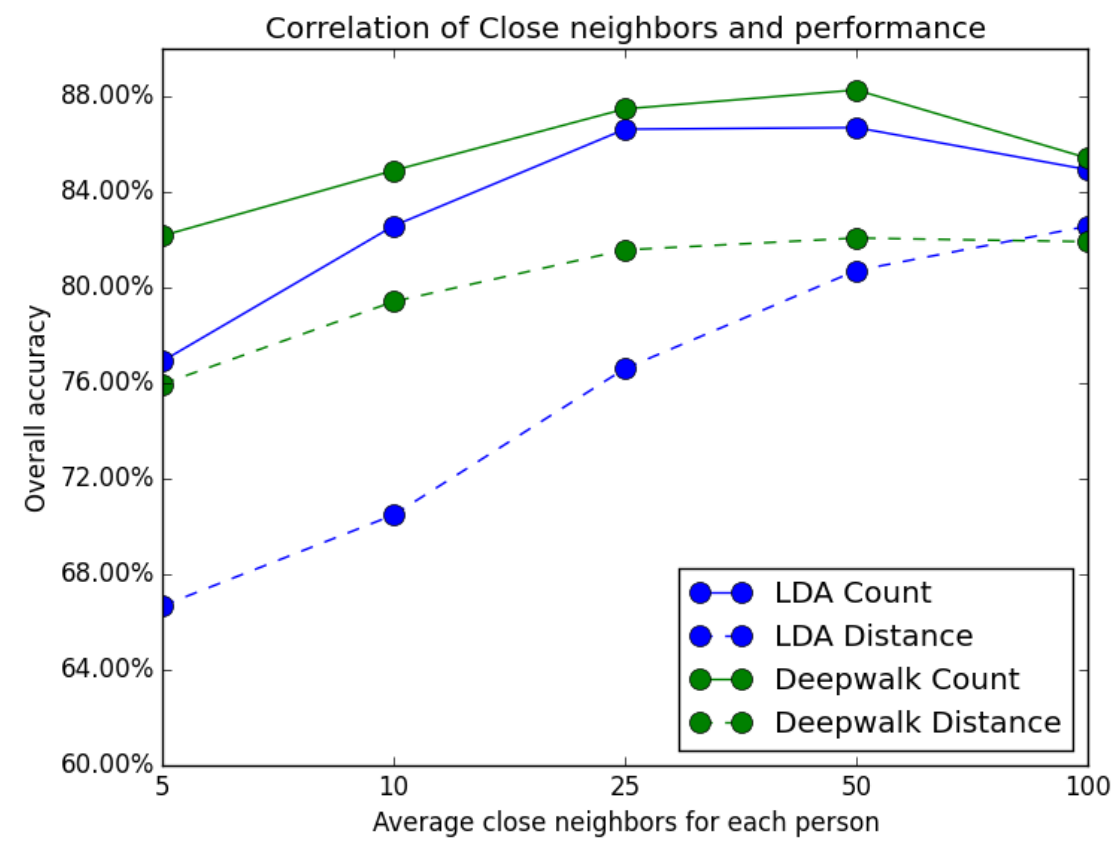

Figure 6: Different definition of close neighbors and corresponding best performance. We experimented with strategies using a fixed number of close neighbors as well as using a comparable number of close neighbors via a distance threshold. Fixed count strategies outperform the distance definition.

people since points in the embedding space are usually evenly distributed.

\subsection{Influence of ranking criteria}

Table 7 provides comparisons between conductance and surprise level metrics. One interesting observation is that the best performance of all conductance measurements happens when creating an average of 100 close neighbors for each point in feature vector space. However, we discovered that surprise level still outperforms conductance even with far fewer close neighbors, indicating that the size of category should be carefully examined during the procedure. 


\begin{tabular}{|c|c|c|c|c|}
\hline & & & \multicolumn{2}{|c|}{ Ranking criteria } \\
\hline & & & Conductance & Surprise level \\
\hline \multirow{6}{*}{ Best } & \multicolumn{2}{|c|}{ Overall Accuracy } & $81.08 \%$ & $88.27 \%$ \\
\hline & \multirow{5}{*}{ Agreement } & 1 st & $36.49 \%$ & $46.47 \%$ \\
\hline & & 2nd & $26.23 \%$ & $24.79 \%$ \\
\hline & & $3 \mathrm{rd}$ & $19.24 \%$ & $14.97 \%$ \\
\hline & & 4 th & $11.11 \%$ & $8.32 \%$ \\
\hline & & 5 th & $6.93 \%$ & $5.44 \%$ \\
\hline \multirow{7}{*}{ Average } & \multicolumn{2}{|c|}{ Overall Accuracy } & $77.63 \%$ & $80.34 \%$ \\
\hline & \multicolumn{2}{|c|}{ Std Deviation } & 0.059 & 0.038 \\
\hline & \multirow{5}{*}{ Agreement } & 1 st & $34.51 \%$ & $37.77 \%$ \\
\hline & & 2 nd & $24.85 \%$ & $23.20 \%$ \\
\hline & & $3 \mathrm{rd}$ & $18.49 \%$ & $18.65 \%$ \\
\hline & & 4 th & $13.03 \%$ & $13.82 \%$ \\
\hline & & 5 th & $9.11 \%$ & $6.57 \%$ \\
\hline
\end{tabular}

Table 7: Performance of each ranking criteria. Best rows describe the highest achieved overall model parameters (distance measurement, close neighbor definition, average count close neighbors and ranking criteria), and corresponding distance distribution in vector spaces. $A v$ erage rows provide information of average accuracy, standard deviation and distribution of average score.

\section{Conclusion}

We have proposed models for constructing feature vectors and measuring the similarity between historical figures, and demonstrated that it works effectively over a representative evaluation environment. We tested our models on approximately 600,000 historical figures from Wikipedia pages, and our Deepwalk embedding yielded an overall accuracy of $88.23 \%$, showing high agreement with human annotated Wikipedia categories. We show that the linear combination of Deepwalk and LDA make the results even better.

These models naturally extend to analyzing figures in different languages, and also to extend to other classes of entities like locations (i.e. cities and 
countries) and organizations (companies and universities). Our methods should be able to identify similar individuals for suggesting friends in social networks, or even matching algorithms pairing up roommates or those seeking romantic partners.

We also proposed models to rank Wikipedia categories according to their descriptive power and demonstrate that descriptive power based on categorical coherence has high correlation with latent representations from feature vectors. The Deepwalk model trained using Wikipedia links yielded the best overall accuracy of $88.27 \%$ in our evaluation, showing a good recovery of importance of Wikipedia categories.

Future work includes parameterizing to capture different tradeoffs between personality, temporal, and topic-based analogies. An inspection of our closest matches suggests that topic-based analogies dominate the nearest matches when considering text only, but more revealing analogies may result from restricting the analyzed word features to particular parts of speech or sentiment polarity.

Finally, we leave it as an open problem to explain or naturally represent the reasons for the observed similarity or analogy. This requires constructing human-interpretable names for the dimensions/topics obtained using our learning procedures, or at least better understanding the meaning of particular strong or overrepresented features, which is an advantage for LDA model. We believe that properly defined weights in combination with different models could better generalize the definition of "similarity" and greatly improve the performance.

\section{Acknowledgments}

480

This research was partially supported by NSF Grants DBI-1355990 and IIS1017181, and a Google Faculty Research Award. We thank Zichao Dai for developing the FameMatch app to evaluate the effectiveness of our category ranking methods. 


\section{References}

485

[1] Y. Chen, B. Perozzi, S. Skiena, Vector-based similarity measurements for historical figures, in: Similarity Search and Applications, Springer, 2015, pp. 179-190.

[2] A. Huang, Similarity measures for text document clustering, in: Proceedings of the Sixth New Zealand computer science research student conference

Natural language processing (almost) from scratch, The Journal of Machine Learning Research 12 (2011) 2493-2537.

q [9] E. H. Huang, R. Socher, C. D. Manning, A. Y. Ng, Improving word representations via global context and multiple word prototypes, in: Proceedings 
of the 50th Annual Meeting of the Association for Computational Linguistics: Long Papers - Volume 1, ACL '12, Association for Computational Linguistics, Stroudsburg, PA, USA, 2012, pp. 873-882.

URL http://dl.acm.org/citation.cfm?id=2390524.2390645

[10] Y. Chen, B. Perozzi, R. Al-Rfou, S. Skiena, The expressive power of word embeddings, in: ICML 2013 Workshop on Deep Learning for Audio, Speech, and Language Processing, Atlanta, GA, USA, 2013.

[11] T. Mikolov, K. Chen, G. Corrado, J. Dean, Efficient estimation of word representations in vector space, arXiv preprint arXiv:1301.3781.

[12] R. Al-Rfou, B. Perozzi, S. Skiena, Polyglot: Distributed word representations for multilingual nlp, in: Proceedings of the Seventeenth Conference on Computational Natural Language Learning, Association for Computational Linguistics, Sofia, Bulgaria, 2013, pp. 183-192.

URL http://www . aclweb.org/anthology/W13-3520

[13] Q. V. Le, T. Mikolov, Distributed representations of sentences and documents, in: Proceedings of the 31st International Conference on Machine Learning (ICML-14), 2014, pp. 1188-1196.

[14] T. Mikolov, I. Sutskever, K. Chen, G. S. Corrado, J. Dean, Distributed representations of words and phrases and their compositionality, in: Advances in neural information processing systems, 2013, pp. 3111-3119.

[15] M. Kim, B.-T. Zhang, J.-S. Lee, Subjective document classification using network analysis, in: Advances in Social Networks Analysis and Mining (ASONAM), 2010 International Conference on, IEEE, 2010, pp. 365-369.

[16] C. Wang, X. Yu, Y. Li, C. Zhai, J. Han, Content coverage maximization on word networks for hierarchical topic summarization, in: Proc. 22nd ACM International Conference on Information and Knowledge Management, ACM, 2013, pp. 249-258. 
[17] A. S. Maiya, R. M. Rolfe, Topic similarity networks: Visual analytics for large document sets, in: Big Data (Big Data), 2014 IEEE International Conference on, IEEE, 2014, pp. 364-372.

[18] B. Perozzi, R. Al-Rfou, S. Skiena, Deepwalk: Online learning of social representations, in: Proceedings of the 20th ACM SIGKDD International Conference on Knowledge Discovery and Data Mining, KDD '14, ACM, New York, NY, USA, 2014, pp. 701-710. doi:10.1145/2623330.2623732 URL http://doi .acm.org/10.1145/2623330.2623732

${ }_{545}$ [19] S. Skiena, C. B. Ward, Who's Bigger?: Where Historical Figures Really Rank, Cambridge University Press, 2013.

[20] R. Kaptein, M. Koolen, J. Kamps, Using Wikipedia categories for ad hoc search, in: Proceedings of the 32nd international ACM SIGIR conference on Research and development in information retrieval, ACM, 2009, pp. $824-825$.

[21] S. Chernov, T. Iofciu, W. Nejdl, X. Zhou, Extracting semantics relationships between Wikipedia categories., SemWiki.

[22] R. Řehůřek, P. Sojka, Software Framework for Topic Modelling with Large Corpora, in: Proceedings of the LREC 2010 Workshop on New Challenges for NLP Frameworks, ELRA, Valletta, Malta, 2010, pp. 45-50. 